\title{
TERAPEUTAS TRADICIONALES ANDINOS EN UN CONTEXTO DE CAMBIO: EL CASO DE CHURCAMPA EN EL PERÚ
}

\author{
Julio Portocarrero ${ }^{1, a}$, Helen Palma-Pinedo $2, a$, M. Amalia Pesantes ${ }^{3, b}$, Gerardo Seminario ${ }^{3, a}$, \\ Claudia Lema $3,4, c$
}

\begin{abstract}
RESUMEN
Objetivos. Describir la diversidad de terapeutas tradicionales, sus vínculos con el sistema de salud oficial y los cambios en sus recursos terapéuticos, en dos comunidades rurales de los andes en Churcampa, región de Huancavelica, Perú. Materiales y métodos. Estudio cualitativo en el que se combinan tres técnicas etnográficas: entrevistas en profundidad, observaciones y acompañamientos a terapeutas tradicionales y personal de salud. Resultados. Se identificaron 58 terapeutas, clasificados como curanderos, parteros y hueseros. La mayoría de terapeutas entrevistados aprendió su labor siendo joven y muy pocos tuvieron un maestro o mentor. La gran mayoría señaló tener reparos para vincularse con los establecimientos de salud más allá de las referencias. No había un trabajo articulado entre personal de salud y terapeutas tradicionales. Sin embargo, cuando sucedía, el personal de salud prefería a aquellos terapeutas que tuvieran la disposición para adecuarse a las necesidades de los servicios de salud. Se encontró que buena parte de los terapeutas estaban cambiando sus prácticas debido a la influencia de las iglesias evangélicas. Ningún terapeuta contaba con discípulos para transmitir sus conocimientos y prácticas. Conclusiones. Existe una gran diversidad de terapeutas tradicionales. Esta diversidad viene disminuyendo, entre otros factores, por el avance de las iglesias evangélicas. Los terapeutas tradicionales refieren a algunos pacientes al establecimiento de salud; mientras que el personal de salud establece relaciones jerárquicas de trabajo con los terapeutas. Se requiere trabajar estas actitudes del personal de salud pues la base de un enfoque intercultural es el trabajo articulado y horizontal entre ambos sistemas.
\end{abstract}

Palabras clave: Medicina tradicional; Etnografía; Chamanismo; Perú (fuente: DeCS BIREME).

\section{TRADITIONAL ANDEAN HEALERS IN THE CONTEXT OF CHANGE: THE CASE OF CHURCAMPA IN PERU}

\begin{abstract}
Objectives. Describe the diversity of traditional healers, their links with the formal health system and changes in their therapeutic resources in two rural Andean communities in Churcampa, in the region of Huancavelica, Peru. Materials and methods. Qualitative study which combined three ethnographic techniques: in depth interviews, observations and accompaniments to traditional healers and health care personnel. Results. 58 traditional healers, classified as healers, midwives and bonesetters were identified. Most healers interviewed learned their work while young and few had a teacher or mentor. The vast majority have objections to linking with health care facilities beyond referrals. There was no coordinated work among health personnel and traditional healers. However, when it happened, health personnel preferred those healers who have the willingness to adapt to the needs of the health services. We found that most of the healers were changing their practices due to the influence of evangelical churches. No healer had disciples to transmit their knowledge and practices. Conclusions. There is great diversity of traditional healers. This diversity is decreasing, among other factors, by the advance of the evangelical churches. Traditional healers refer some patients to the health facility; while health personnel establish hierarchical working relationships with the healers. It is necessary to work on these attitudes of the health care personnel, since the basis of an intercultural approach is to have coordinated and horizontal working relationships between both systems.
\end{abstract}

Key words: Medicine, traditional; Ethnography; Shamanism; Peru (source: MeSH NLM).

Pontificia Universidad Católica del Perú, Lima, Perú.

Dirección General de Epidemiología. Ministerio de Salud. Lima, Perú.

Salud Sin Límites. Lima, Perú.

Centro de Excelencia en Enfermedades Crónicas. Lima, Perú.

a Licenciado en Antropología; ${ }^{\text {a }}$ antropóloga médica, PhD; ' antropóloga médica, magíster

Recibido: : 03-09-14 Aprobado: 07-07-15

Citar como: Portocarrero J, Palma H, Pesantes MA, Seminario G, Lema C. Terapeutas tradicionales andinos en un contexto de cambio: el caso de Churcampa en el Perú. Rev Peru Med Exp Salud Publica. 2015;32(3):492-8. 


\section{INTRODUCCIÓN}

Todo sistema de salud cuenta con agentes encargados de prevenir, tratar y cuidar la salud de las personas, las familias e, incluso, la comunidad en su conjunto (1). Estas personas suelen ser denominados "terapeutas" o "sanadores"; existen distintos tipos dependiendo de su especialización y de los recursos terapéuticos empleados (2). Los mecanismos de legitimación de sus conocimientos y prácticas varían de un sistema médico a otro. Mientras que en algunos sistemas el reconocimiento de sus terapeutas está estandarizado y existen instituciones nacionales y supranacionales que validan sus conocimientos y procedimientos, en otros sistemas su legitimación recae casi exclusivamente en el reconocimiento de la comunidad en la que ejercen (3). Asimismo, mientras que algunos terapeutas han recibido una formación altamente institucionalizada, otros han aprendido su labor sobre la base de la experiencia y la preparación personalizada ${ }^{(4)}$.

Se conoce como terapeutas tradicionales a quienes utilizan una serie de recursos terapéuticos heredados de sus ancestros para prevenir y tratar los padecimientos de los miembros de sus comunidades. Estos recursos terapéuticos pueden ser bastante eclécticos y combinar los conocimientos ancestrales con otros adquiridos a través de su experiencia, de otras tradiciones, e inclusive de prácticas terapéuticas de grupos religiosos. Algunos terapeutas incluyen también elementos de la misma biomedicina promovida desde los establecimientos de salud oficial ${ }^{(5)}$.

En el Perú, es posible encontrar diferentes tipos de terapeutas tradicionales como parteras, sobadores, hueseros, adivinadores, interpretadores de sueños, o vegetalistas ${ }^{(6)}$. Estos pueden ser clasificados por el tipo de padecimientos que tratan, por el repertorio terapéutico que manejan (7) o la dimensión del mundo al que se desplazan o con el que se comunican para tratar. Estas clasificaciones no son siempre útiles para entender la labor que los terapeutas realizan, debido a que, aunque algunos terapeutas se encuentran bastante especializados, otros se mueven entre diferentes prácticas y padecimientos. Frente a esto, se propone una clasificación de menor especificidad basada en dos grandes grupos de terapeutas ${ }^{\left({ }^{8}\right)}$. El primer grupo está conformado por terapeutas que comunican el mundo terrenal con el sobrenatural con fines religiosos y/o terapéuticos ${ }^{(9,10)}$, ellos pueden mantener el control de la moral comunal, predecir el futuro de las siembras, diagnosticar enfermedades, hacer pagos a la tierra o hacer sacrificios a las divinidades para contrarrestar enfermedades ${ }^{(11)}$. Un segundo grupo está compuesto por terapeutas que curan enfermedades o padecimientos a partir del conocimiento de las propiedades curativas de las plantas y otros productos, así como de su preparación ${ }^{(12)}$; estos trabajan también con la energía, los malos sentimientos, los pensamientos o las ideas de las personas ${ }^{(13)}$. Estos terapeutas suelen conocer el funcionamiento de la anatomía y la biología humana, a fin de corregir desequilibrios orgánicos y del pensamiento.

Por lo general, los terapeutas tradicionales viven en un contexto de pluralismo médico en el que coexisten diferentes tipos de curadores, marcos institucionales y repertorios de salud de diversos sistemas médicos ${ }^{(14)}$. Los intercambios entre terapeutas de distintos sistemas no siempre son armoniosos. Las relaciones entre terapeutas pueden basarse en la cooperación o estar a la base de conflictos ${ }^{(15)}$. Incluso, dentro de un mismo sistema pueden existir competencias y enfrentamientos por la búsqueda de reconocimiento o por la interpretación de las enfermedades, entre otros aspectos ${ }^{(16)}$.

En este artículo se presentan los resultados de un mapeo de terapeutas tradicionales realizado en los distritos de Anco y Paucarbamba en Huancavelica, Perú. A partir los hallazgos se propone una reflexión sobre las posibilidades de desarrollar un enfoque intercultural que garantice que la interacción entre los terapeutas de la biomedicina y los tradicionales se dé sobre una base de diálogo y respeto mutuo para un trabajo coordinado (17).

\section{MATERIALES Y MÉTODOS}

Se realizó un estudio cualitativo en el que se combinaron tres técnicas etnográficas: entrevistas en profundidad, observaciones y acompañamiento a terapeutas tradicionales. El trabajo fue realizado por un periodo de dos meses y medio, durante el año 2012, y se concentró en dos distritos de la provincia de Churcampa: Anco y Paucarbamba. Ambos distritos son predominantemente rurales y con una población mayoritariamente quechua hablante. Presentan, además, los más altos niveles de pobreza y los más bajos índices de desarrollo humano de la región y del país.

Las entrevistas se realizaron tanto a personas de la comunidad, como a terapeutas tradicionales y personal de salud; se priorizó a aquellos que tenía más tiempo trabajando en la zona o tenía un mayor conocimiento o contacto con la comunidad

Durante el trabajo de campo se entrevistó, acompañó y registró la actividad de terapeutas tradicionales, de miembros del personal de salud y de representantes de iglesias evangélicas de ambos distritos. Para la identificación de los terapeutas tradicionales se usó la estrategia de bola de nieve, en el que los primeros entrevistados nos llevaban hacia otros en un proceso 
que permitió identificar a más de cincuenta sanadores de diverso tipo. Se incluyó a los terapeutas reconocidos como tales por la población y se buscó tener una muestra diversa que abarcara diferentes especialidades, sexo y nivel de experiencia. Todas las entrevistas fueron realizadas por investigadores de campo bilingües con dominio del quechua y castellano.

Las entrevistas fueron transcritas y traducidas del quechua al castellano por los investigadores de campo; posteriormente, fueron codificadas y procesadas en el software Atlas.ti por el investigador principal del estudio, de acuerdo con las categorías planteadas en el diseño de la investigación. Todos los entrevistados fueron informados previamente de los objetivos de la investigación y se contó con su consentimiento verbal, la entrevista abordó únicamente temas vinculados con su función como terapeutas en su comunidad, evitando abordar temas sensibles, personales o cualquiera que pudiese vulnerarlos.

\section{RESULTADOS}

\section{TERAPEUTAS TRADICIONALES}

Se identificaron 58 terapeutas, clasificados como: curanderos o hampiq, parteros u onqochiq y hueseros. La categoría curanderos incluye una gran diversidad de terapeutas pues se refiere tanto a aquellos que curan con plantas medicinales como a quienes se vinculan con el mundo sobrenatural para tratar a sus pacientes.

Las categorías en la Tabla 1 no son necesariamente excluyentes; buena parte de parteros(as) identificados realizaban otro tipo de prácticas terapéuticas no relacionadas con el embarazo o parto. De igual forma, algunos hueseros ofrecían terapias propias de los clasificados como curanderos.

Tabla 1. Terapeutas tradicionales mapeados por tipo de especialización

\begin{tabular}{lcccccc}
\hline & \multicolumn{2}{c}{ Paucarbamba } & \multicolumn{2}{c}{ Anco } & \multicolumn{2}{c}{ Total } \\
\cline { 2 - 7 } & $\mathbf{N}$ & $\mathbf{( \% )}$ & $\mathbf{N}$ & $\mathbf{( \% )}$ & $\mathbf{N}$ & $\mathbf{( \% )}$ \\
\hline Curandero/a & 19 & $(63)$ & 13 & $(46)$ & 32 & $(55)$ \\
Partero/a & 6 & $(20)$ & 4 & $(15)$ & 10 & $(18)$ \\
Huesero & 5 & $(17)$ & 9 & $(32)$ & 14 & $(24)$ \\
Otros & 0 & $(0)$ & 2 & $(7)$ & 2 & $(3)$ \\
\hline
\end{tabular}

En la Tabla 2 se presenta la proporción de hombres y mujeres identificados como terapeutas (47 y 53\% respectivamente). Existen ciertas diferencias entre las especialidades identificadas y el sexo de los especialistas. Mientras que en el caso de las mujeres existe una proporción similar entre las tres especialidades principales, la mayoría de varones fueron clasificados como hampiq o curandero y en segundo lugar como huesero.

Tabla 2. Tipo de terapeuta tradicional según sexo

\begin{tabular}{lcccccc}
\hline & \multicolumn{2}{c}{ Mujeres } & \multicolumn{2}{c}{ Varones } & \multicolumn{2}{c}{ Total } \\
\cline { 2 - 7 } & $\mathbf{N}$ & $\mathbf{( \% )}$ & $\mathbf{N}$ & $\mathbf{( \% )}$ & $\mathbf{N}$ & $\mathbf{( \% )}$ \\
\hline Curandero/a & 12 & $(39)$ & 20 & $(74)$ & 32 & $(55)$ \\
Partero/a & 9 & $(29)$ & 1 & $(3)$ & 10 & $(18)$ \\
Huesero & 9 & $(29)$ & 5 & $(20)$ & 14 & $(24)$ \\
Otros & 1 & $(3)$ & 1 & $(3)$ & 2 & $(3)$ \\
\hline
\end{tabular}

La mayoría de los terapeutas combinan sus actividades con labores de subsistencia (agricultura, comercio). Los más reconocidos tienen un amplio ámbito de acción abarcando incluso el ámbito regional. Esto parece ser un fenómeno masculino ya que las especialistas mujeres atienden, sobre todo, en su ámbito local. Si bien esta diferencia guarda relación con los roles de género en sociedades rurales, también tiene que ver con la especialidad ejercida. Aquellos que cumplen funciones médico-religiosas, es decir, quiénes pueden tanto curar como adivinar la suerte y hacen pagos para restituir un alma o bendecir una casa, son los que más demanda tienen fuera del ámbito local.

\section{FORMAS DE APRENDIZAJE}

La literatura existente indica que en el Perú, los terapeutas tradicionales presentan tres formas principales de iniciación en determinado saber terapéutico ${ }^{(13)}$.

a. La selección natural/mítica: implica una manifestación hecha por seres superiores que se manifiestan, por ejemplo, a través de sueños, la caída de un rayo u otro fenómeno extraordinario.

b. A partir de un maestro o mentor que puede ser un familiar o no.

c. A partir de la experiencia, observación y reflexión propia.

Según los testimonios recogidos, la mayoría de terapeutas aprendió su labor siendo jóvenes. Muy pocos (solo dos) tuvieron un maestro o mentor, o contaron con un terapeuta tradicional en su familia. La mayoría declara haber aprendido ante la necesidad, al encontrarse con familiares o personas cercanas con alguna enfermedad o padecimiento. Por ejemplo, algunas parteras señalan haber aprendido cuidando de sus hermanas o hijas. Este fenómeno se aprecia también en los sobadores, que desarrollaron sus habilidades al tratar los problemas músculo-esqueléticos de algún miembro de su familia, ganando prestigio cuando sus habilidades trascendieron 
el ámbito inmediato y la comunidad comenzó a reconocer sus competencias como sanador:

Mi esposa desde niña sufría de wiksakuyu, su barriga se había acostumbrado a un lado nomás, incluso, cuando se embarazó se sentía a un costado. Yo mismo tuve que masajearlo y sacudirlo todas las noches, luego vio que se sentía bien y mejoró su salud. Luego una persona se enteró de eso, era un señor que sufría durante muchos años con iguales dolores en la barriga, nadie sabía por qué. Cuando lo trajeron vi que la barriga estaba desparramada (wiksamasta), y le hice masajes hacia el estómago y sacudidos boca abajo de manera que devolvía el estómago a su sitio, esto lo hice por curiosidad. Pero al día siguiente había enviado a su hijo comunicándome que había mejorado y pidiéndome que le vuelva a tratar una vez más. $Y$ así fue que volví a tratar y esta persona se sanó. De allí fui aprendiendo. Esta persona ha ido difundiendo la noticia, y desde ese entonces es que la gente me busca. Yo no busco trabajo, ellos mismos vienen a buscarme, y yo no puedo negarme a ayudar (Terapeuta, Churcampa).

Muchos terapeutas refieren haber aprendido sobre la base de la práctica, buscando referentes más allá de la medicina tradicional de sus comunidades, y recibiendo consejos de diferentes especialistas y no especialistas. Muchos señalan haber probado la efectividad de su repertorio terapéutico sobre la base del ensayo y error así como por los resultados obtenidos en sus pacientes.

Los terapeutas que mencionaron algún maestro o mentor fueron los especialistas que vinculan lo terrenal con lo divino. Sin embargo, ninguno de estos agentes tenía en el momento de la investigación algún aprendiz e indicaron que esto difícilmente iba a ocurrir. El conocimiento que manejan es producto de una revelación que no se da con frecuencia; su preparación no es sencilla pues hay que pasar periodos de tiempo en soledad, aprendiendo y experimentando. Finalmente, refirieron que se debe invertir mucho tiempo y recursos buscando la curación de algunos pacientes, por lo que no es una actividad atractiva para los jóvenes. Lo mismo ocurre con el resto de terapeutas.

La mayoría de los entrevistados indicaron que no existen candidatos a aprendices. Explican que las generaciones más jóvenes no tienen interés en aprender, ni le dan la importancia debida. También se señaló que aunque así lo quisieran no disponen del tiempo ni los recursos suficientes para enseñar sus conocimientos a otra persona.

De acuerdo con los testimonios recogidos, el número de especialistas ha disminuido con el tiempo. A la falta de aprendices y personas "inquietas" (interesadas por aprender) por recibir el conocimiento adquirido se suman las amenazas de algunos trabajadores de los establecimientos de salud y pastores de iglesias evangélicas. Esto hace que muchos sanadores se escondan y disuade a otros de comenzar a ejercer este rol. Una buena proporción de los entrevistados mencionó la presencia de las iglesias evangélicas como un elemento asociado no solo a la reducción sino a la transformación de los terapeutas tradicionales.

\section{TERAPEUTAS Y EVANGÉLICOS}

Una proporción importante de los clasificados por la población como curanderos (un tercio) negó serlo o afirmó no identificarse con el término, describiendo su especialidad según el tipo de repertorio terapéutico utilizado. De este grupo, la gran mayoría eran personas convertidas a la Iglesia Evangélica Pentecostal o a los "Israelitas del Nuevo Pacto Universal". Ellos continuaban considerándose sanadores pero habían cambiado varias de sus ideas y prácticas terapéuticas. Uno de los principales cambios se dio en la etiología de los males. Buena parte atribuía al "diablo" o "demonio" lo que antes era interpretado por ellos mismos como una acción de seres sobrenaturales tradicionales con personalidades complejas que podían hacer tanto el bien como el mal y con los que se debía negociar guardando relaciones de reciprocidad. Este hecho ha transformado parte de su repertorio terapéutico.

Por ejemplo, la mayoría de los sanadores evangélicos señalaba haber abandonado las prácticas de adivinación, diagnóstico y tratamiento a través de divinidades y el desarrollo de "pagos" a la tierra y apus. La mayoría declaró haber adquirido la capacidad de diagnosticar e identificar tratamientos a través de la oración, los cantos religiosos y la comunicación con Dios. Sus terapias actuales incluían cambios en los estilos de vida de los pacientes como dejar de tomar y fumar o acudir con más frecuencia a la iglesia. Algunos de estos especialistas evangélicos habían adoptado al "requinto" dentro de su repertorio terapéutico, esto consiste en tratar el padecimiento de una persona que sufre debido a agentes malignos, expulsando el mal de su cuerpo a través de rezos y alegatos en nombre de dios.

\section{ACCIONES DEARTICULACIÓN ENTRE TERAPEUTAS TRADICIONALES Y PERSONAL DE SALUD OFICIAL}

En el Perú, un reto pendiente es el trabajo articulado entre los terapeutas tradicionales y el servicio de salud oficial. Al indagar sobre el tema, encontramos diferentes posiciones. La mayoría de terapeutas tradicionales entrevistados considera que su conocimiento y terapéutica no es excluyente del servicio de salud oficial, y que pueden 
Tabla 3. Formas de articulación con servicios de salud oficial según tipo de terapeuta

\begin{tabular}{lccccc}
\hline & $\begin{array}{c}\text { Conversación } \\
\text { con el personal } \\
\text { de salud }\end{array}$ & $\begin{array}{c}\text { Ayudan o enseñan } \\
\text { al personal de } \\
\text { salud }\end{array}$ & $\begin{array}{c}\text { Han atendido } \\
\text { pacientes dentro del } \\
\text { puesto }\end{array}$ & $\begin{array}{c}\text { Han realizado } \\
\text { actividades conjuntas } \\
\text { con el personal }\end{array}$ & $\begin{array}{c}\text { Han realizado } \\
\text { referencia al centro }\end{array}$ \\
\hline Parterala & No & No & Sí en 2 casos & Sí en 2 casos & Sí \\
Curandera/a & No & No & No & No & Sí \\
Huesero & No & No & No & No & Sí \\
\hline
\end{tabular}

actuar en paralelo o de manera complementaria. Esto se evidencia al observar que la mayoría de terapeutas ha realizado en el último año algún tipo de referencia hacia los centros de salud (Tabla 3).

La mayoría de terapeutas señaló tener reparos para vincularse con los establecimientos de salud más allá de las referencias. Esto tiene que ver, en parte, con la forma en que se han relacionado previamente con el servicio. Por ejemplo, un trabajador de salud de Anco recordó que hace un par de años uno de sus colegas amenazó a una curandera diciéndole "que no se metiera porque sino, la denunciarían." Eventos como este amedrentan a los terapeutas.

Para el personal de salud entrevistado los casos de colaboración deben darse en sus términos. El personal de salud considera que son ellos quienes deben definir cuándo y cómo deben participar los terapeutas, manteniendo una relación vertical.

Sí, ellos nos apoyan bastante, pero hay que poner en claro algunas cosas, limitantes hasta dónde puede ir su ayuda; en eso si soy muy enfática con ellos, en cuanto a gestantes y sí me ha dado resultados. Pero si es posible, porque son personas accesibles que no son cerrados (...) hemos tenido varias experiencias y son ya, años de trabajo (trabajador de salud de Anco).

Encontramos que los casos de articulación experimentados en la zona parecen ser de carácter unilateral:

Con el señor Víctor hemos conversado y al menos hemos llegado a un acuerdo. A veces, las gestantes van en trabajo de parto y el Sr. Víctor mediante el pulso les dice si falta o no para el parto. Entonces hemos conversado con él para que ya no las devuelva a su casa, porque muchas de ellas primero van donde él, sino que las envíe directamente al puesto de salud. Y que si va a hacer manteo a las gestantes, porque ellas siguen creyendo que eso es bueno para ellas, que lo haga en forma muy suave y ya no en las últimas semanas de embarazo. Y que si se presentan casos de hemorragia así sea muy poquito que no se atreva ya a tocarlos y que las mande inmediatamente al establecimiento de salud (trabajador de salud de Paucarbamba).
El personal habla de terapeutas tradicionales cerrados y de los que no lo son. Esto implica dos elementos: el primero es la apertura hacia el conocimiento biomédico, el segundo es su disposición a obedecer al personal de salud. Encontramos en nuestro análisis que los terapeutas menos cerrados son las(os) parteras(os) y hueseros; y los más cerrados son los curanderos que se comunican con los apus y aquellos que participan de los cultos evangélicos.

El personal de salud considera que no sería adecuado trabajar con cualquier terapeuta, ni de cualquier especialidad. Esto tiene que ver con las prioridades del servicio, la inocuidad de los tratamientos tradicionales y la voluntad de adecuación de los terapeutas. Por ejemplo, existe una fuerte aceptación de la adecuación de la atención del parto. Esto, debido a que la atención del parto tradicional fue desde inicios del 2005 especialmente impulsada por distintos actores del Estado y la sociedad civil en la zona de investigación. Churcampa estuvo muy vinculada al desarrollo de la "Norma Técnica Nacional para la Atención del Parto Vertical", lo que ha facilitado su incorporación en el trabajo cotidiano del personal de salud. Se encontró que el personal de salud está especialmente interesado en conocer las propiedades de las plantas utilizadas por los terapeutas y la experiencia de las parteras. Para el personal de salud, la articulación con los sanadores sirve, sobre todo, para acercar a los pacientes, pero no para considerar ni valorar su conocimiento o eficacia terapéutica.

\section{DISCUSIÓN}

El presente estudio muestra un sistema tradicional que atraviesa por una serie de procesos transformadores, entre los cuales la presencia de las creencias evangélicas son los que tienen mayor influencia. Estamos ante un escenario de cambio en el que cada vez se utilizan menos los rituales de comunicación con seres sobrenaturales y hay variación en los recursos terapéuticos que manejan. Como se ha visto en otros contextos, la paulatina reducción de terapeutas tradicionales afecta la forma cómo la población entiende su salud y los itinerarios para solucionar sus dolencias ${ }^{(2)}$.

Este estudio ha sido realizado en dos distritos andinos del Perú, lo cual limita la capacidad para extrapolar 
nuestros resultados a otros países de la región andina. Sin embargo, estudios en otros países latinoamericanos, como México, muestran que los sistemas de salud indígenas siguen vigentes en zonas urbanas ${ }^{(17)}$.

Se evidencia la gran diversidad de terapeutas y su marginación del sistema de salud oficial. Encontramos que la mayoría de los terapeutas tradicionales tiene reparos en relacionarse con el sistema de salud biomédico, pero ponen en práctica algunas formas de interacción, como la referencia de pacientes hacia los establecimientos de salud. No se encontraron testimonios de referencias desde el sistema de salud oficial hacia el tradicional, que daría indicios de un enfoque intercultural por atender la salud de la población.

En el Perú, en el 2006, la Dirección General de Promoción de la Salud (DGPS) del Ministerio de salud (MINSA) publicó el documento técnico "Enfoque de derechos humanos, equidad de género e interculturalidad en salud: marco conceptual" que establece el uso de un enfoque intercultural en los servicios de salud implica generar condiciones para el trabajo articulado entre terapeutas tradicionales y personal de salud (18).

Como muestra este trabajo, una de las barreras para la implementación de un trabajo articulado entre sistemas de salud es el poco conocimiento que el personal tiene de la cantidad y diversidad de terapeutas que ejercen en su zona de trabajo y de las prácticas terapéuticas que usan (19). Si el MINSA busca consolidar el uso de un enfoque intercultural en los servicios de salud, un primer paso es promover el desarrollo de mapeos como el presentado en este estudio y propiciar encuentros que faciliten el aprendizaje mutuo entre terapeutas y personal de salud.

Agradecimientos: a Médicos Mundi Navarra, a los terapeutas de las zonas visitadas, a los pobladores que brindaron su tiempo y apoyo. MAP tiene una beca posdoctoral del Consejo Nacional de Ciencia y Tecnología (CONCYTEC). A Fernando Carbone y Marcela Velásquez.

Contribuciones de autoría: JP realizo la concepción y diseño del artículo y con HPP participaron en la obtención de resultados, análisis e interpretación de datos, redacción del artículo y aprobación de su versión final. MAP, GS y CL participaron en la redacción y revisión crítica del artículo, además $\mathrm{CL}$ obtuvo el financiamiento para el trabajo.

Fuentes de financiamiento: el presente artículo es producto de una investigación solicitada, diseñada y financiada por el proyecto "Un modelo integral e incluyente bajo un concepto de derecho a la salud", ejecutado en el Perú por Medicus Mundi Navarra y Salud Sin Límites Perú y financiado por la Unión Europea y el Gobierno de Navarra.

Conflictos de interés: los autores declaran no tener conflictos de interés

Descargos de responsabilidad: los puntos de vista expresados en esta publicación, no necesariamente reflejan los puntos de vista de la Dirección General de Epidemiología.

\section{REFERENCIAS BIBLIOGRÁFICAS}

1. Sobo E. Theoretical and Applied Issues in Cross-Cultural Health Research, En: Ember M, Ember CR. Encyclopedia of Medical Anthropology: Health and illness in the World's Cultures. New York: Springer; 2004. p. 3-11.

2. Stacy Leigh P. "Found in Most Traditional Societies": Traditional Medical Practitioners between Culture and Development. In: Cooper F, Packard $\mathrm{R}$, ed. International Development and the Social Sciences. Berkeley: University of California Press; 1997. p. 259-90.

3. O'Connor B. Healing Traditions: Alternative medicine and the health professions. Philadelphia: University of Pennsylvania; 1995.

4. Kleinman A. Concepts and a model for the comparison of medical systems as cultural systems. Soc Sci Med. 1978; 12(2B):85-95.
5. Singer M, Davison L, Gerdes G. Culture, critical theory, and reproductive illness behavior in Haiti. Med Anthropol Q. 1988;2(4):370-85.

6. Delgado Sumar H. Los especialistas en la medicina tradicional. Lima: Instituto Nacional de Medicina Tradicional; 2000.

7. Delgado Sumar H. Las técnicas curativas en el mundo andino: el diagnóstico de las enfermedades. Lima: Instituto Nacional de Medicina Tradicional; 2000.

8. Delgado Sumar H. La pluralidad de la medicina tradicional. Lima: Instituto Nacional de Medicina Tradicional; 2000.

9. Bastien JW. Qollahuaya-Andean body concepts: a topographical-hydraulic model of physiology. Am Anthropol. 1985;87(3):595-611.
10. Reichel E. Cosmology, worldview and gender-based knowledge systems among the Tanimuka and Yukuna (Northwest Amazon). 1999;3(3):213-42.

11. Polia Meconi M. Despierta, remedio, cuenta...: adivinos y médicos del Ande. Lima: Fondo Editorial de la Pontificia Universidad Católica del Perú; 1996.

12. Reichel-Dolmatoff G. Rainforest Shamans: Essays on the Tukano Indians of the Northwest Amazon. Totnes: Themis Books; 1997.

13. Delgado Sumar H. Las técnicas curativas en el mundo andino: el tratamiento de las enfermedades. Cuadernos de Medicina Tradicional 7. Lima: Instituto Nacional de Medicina Tradicional; 2000.

14. Ramírez Hita S. Donde el viento llega cansado. Sistemas y prácticas de salud 
en la ciudad de Potosí. 2da Ed. La Paz: Editorial Gente Común; 2010.

15. Castañeda Camey X, García Barrios C, Romero Guerrero X, Nuñez-Urquiza RM, Gonzalez Hernández D, Langer Glass A. Traditional birth attendants in Mexico: advantages and inadequacies of care for normal deliveries. Soc Sci Med. 1996;43(2):199-207.

16. MenéndezE. La enfermedadylacuración. ¿Qué es la medicina tradicional? Revista Alteridades 1994;7:71-83.
17. Berenzon-Gorn S, Ito-Sugiyama E, Vargas-Guadarrama LA. Enfermedades y padeceres por los que se recurre a terapeutas tradicionales de la Ciudad de México. Salud Publica Mexico. 2006;48(1):45-56.

18. Perú, Ministerio de Salud. Documento técnico: marco conceptual de los derechos humanos, equidad de género e interculturalidad en salud. Lima: MINSA; 2006.

19. Cáceres Chalco E. El curandero: un especialista andino. Grupo Pachawaray,
No. 11. Cusco: Grano de Arena; 1999. p. 10-2.

Correspondencia: Julio Portocarrero Gutiérrez.

Dirección: Facultad de Ciencias Sociales, Pontificia Universidad Católica del Perú, Av. Universitaria 1801, San Miguel, Lima 32, Perú Correo electrónico:juliocesarportocarrero@ hotmail.com

\section{http://twitter.com/rpmesp}

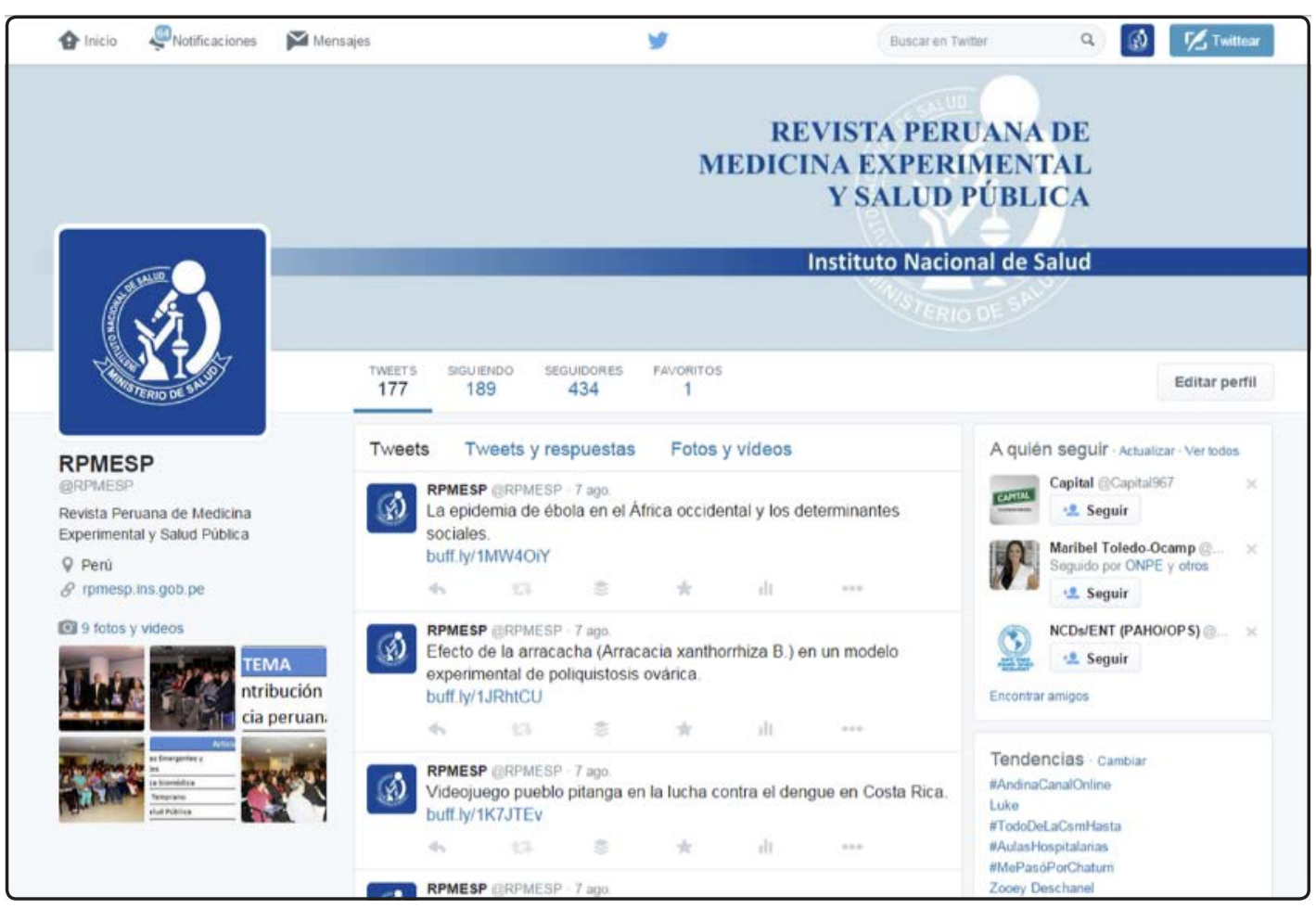

